\title{
Mechanical complications of shunt for hydrocephalus
}

\author{
Aline Saré de Melo, MD1 \\ Received: 01 March 2020 / Published: 22 March 2020
}

\begin{abstract}
Introduction: Hydrocephalus is the pathology most frequently addressed and studied in pediatric neurosurgery. Over 15,000 new cases are diagnosed each year. Although it is the most com-mon procedure performed by pediatric neurosurgeons, the shunt implant for drainage of cerebrospinal fluid (CSF) also presents the highest rate of failure and replenishment. The last 50 years have been marked by important advances in quality of these implants. How-ever, little has changed regarding the prevalence of these failures. We will comment here the main mechanical failures of the shunt: disconnection, migration, fracture and scrap-ing of the catheter, hollow visceral perforation and distal catheter knot.

Methods: Literature review about the main causes of mechanical failure in shunts

Conclusion: Shunt dysfunction is still very common, despite technological advances in the treatment of hydrocephalus. Mechanical causes of shunt dysfunction correspond to about $30 \%$ of the reasons for changing or overhauling valves. Most of these occur due to surgical problems. Therefore, safe measures reduces these events their clinical features.
\end{abstract}

Key words: Hydrocephalus; pediatric neurosurgery; cerebrospinal fluid shunt; mechanical complications

\footnotetext{
1 Section of Pediatric Neurosurgery, Department of Surgery Americas Medical Group, Section of Neurosurgery, Ipanema Federal Hospital

To whom correspondence should be addressed: Aline Saré de Melo, MD [E-mail: alinesare@gmail.com]
}

Journal homepage: www.sbnped.com.br

\section{Introduction}

The history of hydrocephalus treatment with valves evolved very little until the 1960s. However, in the last 50 years [1] several technological innovations of design and placement of shunt have changed the behavior and natural history of this treatment [2].

Hydrocephalus is a complex pathology, with several manifestations related to different etiologies, such as congenital malformations, trauma, tumors, infection and hemorrhage. The cause of hydrocephalus is proven to be a statistical factor for ventricular shunt dysfunction [4]. Pediatric and adolescent patients with hydrocephalus associated with CNS development abnormalities, present a higher risk of complications after CSF drainage surgery, compared to patients without CNS related abnormalities $[3,9,10]$. Those with CNS disorders show a higher risk of life, with a more severe clinical presentation.

Shunts of cerebrospinal fluid (CSF) are among the most life-sustaining medical devices prone to failure, implanted in modern medical practice, with failure rates of 30 to $40 \%$ in 1 year and approximately $50 \%$ in 2 years in pediatric patients.

Since the first peritoneal drainage device created in 1898 by Fergusson, many other modern shunt models have been launched, based on mistaken calculations of CSF pro-duction, absorption and circulation. Despite the rational or often "more physiological" functioning of these devices, complications continue to occur at similar rates, which re-duced the initial enthusiasm in these new valves. As the improvements in the derivation project seem very limited, there was incentive and space for studies on endoscopic treat-ment. Today, undoubtedly, this technique is the great hope and revolution in the treatment of most cases of hydrocephalus. However, there will always be room for derivations, since the use of endoscopy is limited in some etiologies.

The literature is abundant in comparative studies for the effectiveness of the most used devices (such as the Cordis Orbis Sigma and Medtronic PS Medical 
Delta valves) [5]. Surprisingly, no relevant differences were found in the failure rates of these and other less complex systems $[6,7,8]$.

\section{Material and Methods}

PubMed US National Library of Medicine; National
Institutes
http://www.ncbi.nlm.nih.gov/pubmed/) with the following groups of keywords: dysfunction $\mathrm{OR}$ blockage AND hydrocephalus; shunt complications AND hydrocephalus AND shunt AND mechanical complications. A total of 86 articles were retained for analysis (English and French). This review will focus on the mechanical causes of shunt failure, thus excluding infections and obstructions.

\section{Discussion}

Derivation failures are frequent and can be clinically present in many ways, according to the patient's age, infants and young children may have an increase in head circumfer-ence, bulging fontanelle, Parinaud's syndrome. The story usually includes nausea, vom-iting, irritability or altered consciousness. Seizure, diplopia, weakness or visual loss may also be present. During the neurological exam, it is possible to find papilledema, cranial nerve palsy (CN VI), hyperactive reflexes and ataxic gait. Sometimes it is possible to find signs of shunt dysfunction through the palpation of the vale reservoir. The accumulation of fluid in the ventricular insertion portion or in the subcutaneous part of the distal cathe-ter pathway may suggest its failure, disconnection or fracture.

The studies on shunt dysfunctions are consistent in the general incidence of failure after the initial insertion [12]. Most present approximately $40 \%$ in 1 year and $50 \%$ in the second year after insertion. Long term follow-up data from the Shunt Design Trial demonstrated an overall shunt survival of $41 \%$ over 4 years. In another study up to $50 \%$ in first year of life and up to 4 to $5 \%$ per year thereafter, and as high as $81 \%$ after 12 years [12].

Although failure rates are high, some predictive factors are known. Tuli et al. found that the patient's age at the time of the initial shunt insertion and the time interval since the previous surgical revision were important factors for repeated shunt failures. Similar re-sults were found in other prospective studies (Endoscopic Shunt Insertion Assay, Fears).

\section{Mechanical Shunt Failure}

Failures of many types of shunt occur at different times after insertion surgery. Infection normally occurs in the first 6 months and is directly related to the surgical procedure in its majority [13]. The obstruction of the system (proximal, distal or valve catheter) can occur at any time after its placement. Among the mechanical causes of dysfunction, the loss, disconnection or migration of components of the catheter shunt may occur earlier. Fractures, perforation of the hollow viscus by the peritoneal catheter or loops and knots usually oc-cur in the long term.

\section{Disconnection}

Several shunts models are presented in two or three separate parts, which are surgically connected. Disconnection at these points is due to errors in the surgical approach and normally occurs early.

Disconnections in the system interrupt the flow of CSF, causing generally insidious symptoms of hydrocephalus. A subcutaneous CSF collection can be seen around the proximal point of disconnection. Imaging exams, such as ultrasound, cross-sectional to-mography and resonance can easily identify such collections. Due to the risk of sudden neurological worsening, disconnection should be treated surgically, as an emergency, even in oligiosymptomatic patients [14]. The technique may vary from simple reconnection of parts to replacement of part or the entire system. Evaluation of possible points with tension and connection with firm knots, should be reviewed.

Some authors recommend the "assembly" of the system on a separate table before im-plantation, which is not routine in our service. Today in the market, we find systems with reduced to one or none connections. The lower need for ties, dramatically reduces the separation rate. The unishunt (Codman / Johnson \& Johnson, Raynham, MA) does not require surgical assembly, but due to the specific technique for its implant, it has not yet had adequate acceptance among the neurosurgical community.

\section{Migration}

It is the phenomenon by which a properly placed catheter moves from its original appro-priate location to a position where cerebrospinal fluid no longer drains or drainage is se-verely impaired. As the child grows up, the catheter tips may come out of the ideal position, blocking CSF drainage. This phenomenon may occur both with the proximal cathe-ter, which is removed from the ventricle, and with the distal catheter, which is removed from the cardiac atrium, the pleural space or the peritoneum.

Selection of the type of catheter has been implicated in its migration. Those with a 900 angle of entry into the skull tend to resist migration better. Fixing the distal catheter to fixed structures as entry into the peritoneum in the VPS (ventriculo peritoneal shunt) or cervical subcutaneous tissue in the VAS (ventriculo atrial shunt) may create tension during the child's growth and favor the catheter's migration. The patient may present clinical symptoms similar to obstruction due to the loss of drainage capacity. 
Additional clinical findings may include a palpable change in valve position or fluid col-lection around the shunt. Should be an avoidable problem, when potential fixation sites that can pull the valve and the ventricular catheter, as connection points. It can be eliminated with the placement of a new peritoneal catheter (13). The treatment of this complication is limited to the repositioning of the ventricular catheter and proximal fixation of the valve in order to avoid traction of the system with the child's movement and growth [14].

\section{Fracture}

A major cause of fracture is calcification of the catheter. Dysfunction of the shunt for this reason is not frequent. Very few cases of shunt rupture and calcification have been re-ported in the literature, and these conditions generally occur as a long-term complication of VPS (Figure 1). In these cases, in addition to the presence of symptoms of shunt dysfunction with intracranial hypertension, we may also find pain in the neck and chest wall along the bypass, with limited neck movements as a result of tying the bypass tube. The degradation of the silicone material and the deposit of calcium and other minerals in the shunt catheters may lead to fracture or migration of the system. Shunt calcification can cause dysfunction in two ways, either by disconnection or obstruction.

These are mainly due to the fixation of the catheter. Barium sulfate mixed with silicone during the bypass tube manufacturing process can accelerate late complications due to the formation of cracks in the catheters. Calcification was observed mainly in catheters impregnated with barium. The introduction of silicone-coated bypass tubes can reduce the values of this condition [15].

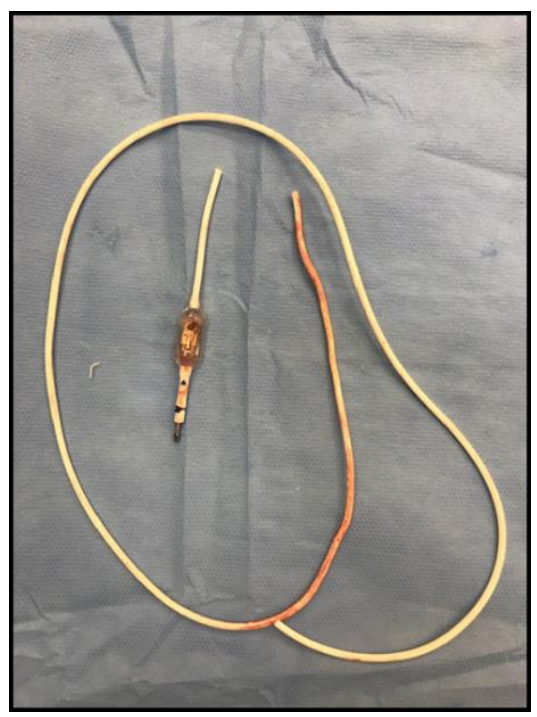

Figure 1 - Catheter extracted from a 14-year-old patient, implanted since birth. The patient presented intermittent headache and vomiting associated with a small subcutaneous collection in the cervical region.

\section{Hollow viscera perforation}

There are many reports on perforation of the intestinal loop or other abdominal structure through the distal catheter. This is usually a late complication, but it can also occur in the short term, as in the case of neonates or children with neurological pathology undergoing other abdominal interventions. The presence of adhesions and an abdominal inflammatory process, associated with chronic constipation, common in this population, favor the occurrence of follow visceral perforation.

\section{Loop or knot in the distal catheter}

The knot in the peritoneal catheter is considered a rare cause of mechanical dysfunction of ventricle peritoneal shunt. There are few reports in literature of this complication. The knot usually occurs in the distal portion of the catheter and its exact mechanism has not yet been clarified [16]. Several theories have been suggested, such as abdominal cavity capacity, characteristics of the catheter, surgical technique, catheter that is too long, in-creased peristalsis, presence of adhesions and meteorism may be related.

When the knot occurs around the intestine, bowel obstruction and ischemia may occur. Mohinda and Sharma [17] reported a knot around the intestine, but the patient was asymptomatic. Being an incidental finding, this may be a silent complication. Some authors refer to intense peristalsis as a triggering factor. Others suggest that the presence of intestinal obstruction and formation of intra-abdominal cyst could be causes of the knot.
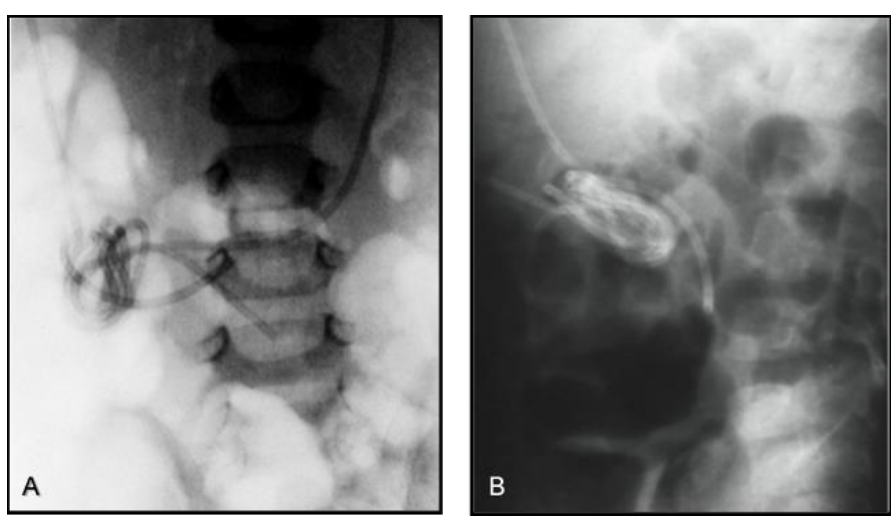

Figure 3 - A. Simple abdominal X-ray in AP, showing the entrapped peritoneal catheter, inside the intraperitoneal cavity. B. Simple abdominal X-ray in profile showing the folding of the peritoneal catheter.

\section{Conclusions}

Pediatric hydrocephalus is a prevalent condition with multiple causes. Shunt dysfunction is still very common, despite technological advances in the treatment of hydrocephalus. Most studies indicate the 

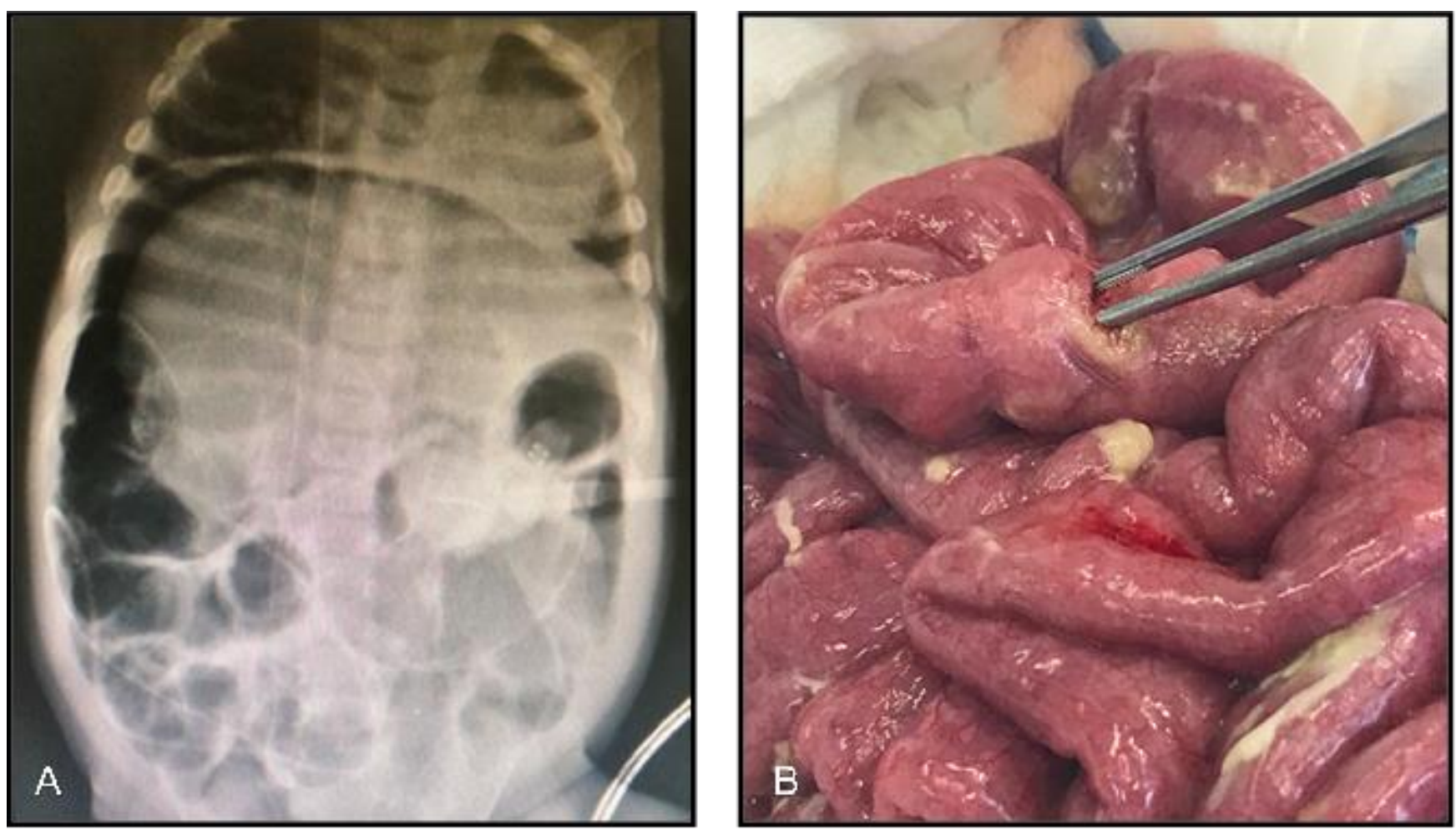

Figure 2: A. Large pneumoperitoneum in a 4-month-old infant, due to perforation of the intestine by a distal catheter. B. Surgical image of the perforation hole.

etiology of hydrocephalus as the main determining factor for mechanical failure and system dysfunction and the patient's age at the time of the initial shunt insertion. New projects should focus on the etiology of the disorder, as therapies may need to be adapted to the etiology to obtain the best overall survival and patient out-come. We are facing the challenge of identifying these factors that contribute to failures, in order to offer patients a better choice. According to the literature, the type of valve or its model is not considered as a risk factor for shunt failure, time to its first revision or even the number of its revisions.

In view of the short-term reality, which has little promise in the effective reduction of shunt complications, third ventriculostomy is an effective tool. Its indication and success are related to the underlying causal pathology. If "the best shunt is no shunt", the neurosurgeon community has been increased the application those treatments (ETV) $[17,18]$.

\section{Conflict of interest}

The authors report no conflict of interest concerning the materials or methods used in this study or the findings specified in this paper.

\section{References}

1. Nulsen F, Spitz E. Treatment of hydrocephalus by direct shunt form ventricle to jugular vein. Surg Forum. 1952;399-403

2. Drake J, Kestle J, Tuli S. CSF shunts 50 years on past, present and future. Child's Nervous System. 2000;16(10-11):800-804.

3. Raguz M, Brcina N, Marinac D, Gjurasin M. Influence of congenital central nervous system malformations on postoperative complications following placement of cerebrospinal fluid shunts in pediatric and adolescent patients. Wiener klinische Wochenschrift. 2013;126(1-2):2-8.

4. Piatt, Jr. J, Carlson C. A Search for Determinants of Cerebrospinal Fluid Shunt Survival: Retrospective Analysis of a 14-Year Institutional Experience. Pediatric Neurosurgery. 1993;19(5):233-242.

5. Sainte-Rose C, Hooven MD, Hirsch JF. A new approach to the treatment of hydrocephalus. J Neurosurg. 1987; 66:213-226.

6. Sainte-Rose C. Shunt Obstruction: A Preventable Complication?. Pediatric Neurosurgery. 1993;19(3):156-164.

7. Riva-Cambrin J, Kestle J, Holubkov R, Butler J, Kulkarni A, Drake $\mathrm{J}$ et al. Risk factors for shunt malfunction in pediatric hydrocephalus: a multicenter prospective cohort study. Journal of Neurosurgery: Pediatrics. 2016;17(4):382-390.

8. Neiter E, Guarneri C, Pretat P, Joad A, Klein O, Marchal J. Sémiologie d'un dysfonctionnement de 
dérivation ventriculo-péritonéale chez l'enfant - mise au point. Neurochirurgie. 2016:53-59.

9. Notarianni C, Vannemreddy P, Maldito G, Bollam P, Wylen E, Willis B. Congenital hydrocephalus and ventriculoperitoneal shunts: influence of etiology and programmable shunts on revisions. J Neurosurg Pediatrics. 2009;4:547-552.

10. Raguz $M$, Brcina $N$, Marinac $D$, Gjurasin $M$. Influence of congenital central nervous system malformations on postoperative complications following placement of cerebrospinal fluid shunts in pediatric and adolescent patients. Wiener klinische Wochenschrift. 2014 Jan 1;126(1-2):2-8.

11. Lazareff JA, Peacock W, Holly L, Ver Halen J, Wong A, Olmstead C. Multiple shunt failures: an analysis of relevant factors. Child's nervous system. 1998 Jun 1;14(6):271-

12. Rahalkar MD. Complications of Cerebrospinal Fluid Diversion (Shunt) Catheters: A Pictorial Essay. Indian Journal of Neurosurgery. 2018 Mar;7(01):05876.

13. Browd SR, Ragel BT, Gottfried ON, Kestle JR. Failure of cerebrospinal fluid shunts: part I: obstruction and mechanical failure. Pediatric neurology. $2006 \mathrm{Feb}$ $1 ; 34(2): 83-92$.
14. Hanak BW, Bonow RH, Harris CA, Browd SR. Cerebrospinal fluid shunting complications in children. Pediatric neurosurgery. 2017;52(6):381-400.

15. Salim AD, Elzain MA, Mohamed HA, Zayan BE. Shunt tube calcification as a late complication of ventriculoperitoneal shunting. Asian journal of neurosurgery. $2015 \mathrm{Jul} ; 10(3): 246$.

16. Pereira CU, Morais RM. Complicação Rara de Mau Funcionamento de DVP. JBNC- Jornal Brasileiro de Neurocirurgia. 2015;26(1):72-4.

17. Mohindra S, Sharma M. Spontaneous knotting of peritoneal catheter: a report of an asymptomatic patient. Journal of pediatric neurosciences. 2012 May;7(2):151.

18. Iglesias S, Ros B, Martín Á, Carrasco A, Segura M, Delgado A, Rius F, Arráez MÁ. Surgical outcome of the shunt: 15-year experience in a single institution. Child's Nervous System. 2016 Dec 1;32(12):2377-85. 19. Beuriat PA, Puget S, Cinalli G, Blauwblomme T, Beccaria K, Zerah M, Sainte-Rose C. Hydrocephalus treatment in children: long-term outcome in 975 consecutive patients. Journal of Neurosurgery: Pediatrics. 2017 Jul 1;20(1):10-8. 